\title{
I mplementing an evidence-based practice protocol for prevention of catheterized associated urinary tract infections in a progressive care unit
}

\author{
Kathleen Revello ${ }^{1}$, Ana-Maria Gallo² \\ 1. Sharp Grossmont Rehabilitation Center, Sharp Grossmont Hospital. United States. 2. Nursing Education, Research, and \\ Professional Development, Sharp Grossmont Hospital, United States.
}

Correspondence: Kathleen Revello. Address: Sharp Grossmont Rehabilitation Center, Sharp Grossmont Hospital, La Mesa, CA. 91942. Email: Katie.revello@Sharp.com

Received: May 10, 2012

Accepted: J une 28, 2012

Published: January 1, 2013

DOI : $10.5430 /$ jnep.v3n1p99

URL: http://dx.doi.org/10.5430/jnep.v3n1p99

\begin{abstract}
Catheter associated urinary tract infections (CAUTI) are common, painful, and potentially life threatening to hospital patients. Nurses implemented an evidence-based project to determine if a new protocol would decrease the incidence of CAUTIs. The project included a point prevalence study, healthcare team education and a prompt communicating catheter insertion date, time and removal recommendations. This project was successful as evidenced by post data collection showing CAUTI rate decreases of 1.1/1000 catheter days and a device duration decrease of 0.2 device days/patient days.
\end{abstract}

\section{Key words}

Evidence-based practice protocol, Catheterized associated urinary tract infections, Progressive care unit

\section{Introduction}

Studies have shown that Urinary Tract Infections (UTIs) are the most common hospital acquired infections and that 80 percent of UTIs are a result of indwelling urinary catheters ${ }^{[1]}$. Symptoms of a UTI can range from mild (fever, urethritis, and cystitis) to severe (renal scarring, acute pyelonephritis, and bacteremia). Left untreated, these infections can lead to urosepsis and death ${ }^{[2]}$. These infections are painful for patients and costly for hospitals due to increased length of stay and use of additional resources.

As of 2010, the Center for Medicare and Medicaid (CMS) no longer reimburses hospitals for the additional costs of treating nosocomial infections such as hospital acquire urinary tract infections. In May of 2011, the Joint Commission announced a new national patient safety goal that requires hospitals to implement an evidence-based practice program to prevent catheter associated urinary tract infections (CAUTIs) by January 1, 2013. It was essential that a hospital implement the most current evidence supporting the successful reduction of these infections. The purpose of this evidence-based practice project was to decrease the CAUTI rate in the progressive care unit. A progressive care unit is a medical/surgical unit that provides telemetry monitoring. 


\section{Background}

The indwelling catheter has been used since the mid-1930s. Currently, the indwelling catheter continues to be the most commonly used medical device for the management of urinary incontinence ${ }^{[3]}$. Although the use of the indwelling catheter is needed for therapeutic and diagnostic purposes, over use has become common practice. The most common reasons for failure to discontinue urinary catheters in a timely manner included: poor patient mobility, urinary incontinence, or lack of prioritization due to nurse's workload and minimal understanding of the risks of indwelling urinary catheters ${ }^{[4]}$.

The National Healthcare Safety Network (NHSN) defines symptomatic CAUTI as a UTI that occurs in a patient who had an indwelling urethral urinary catheter in place within the 48 hour period before the onset of the UTI. The symptoms may include fever, urgency, frequency, dysuria, suprapubic tenderness, and a positive urine culture with no more than two species of organisms.

This project took place at a 536 bed, Magnet designated, tertiary care hospital in San Diego County. Magnet designation is given by the American Nurses Credentialing Center (ANCC) to recognize hospitals that provide quality patient care, nursing excellence, and innovations in nursing practice. At this hospital, removal of the catheter in a timely manner was not a focus for medical patients. Surgical patients had the advantage of preprinted orders for catheter removal on post-operative day three in compliance with the Surgical Care Improvement Project (SCIP-Inf-9) initiatives. Improvement to remove catheters in a timely manner in medical patients was needed.

CAUTI rates have been reported nationally by the ICUs exclusively and have been used as benchmarks in measuring quality of nursing care for a hospital. This hospital's surgical ICU has 24 beds and has patients with abdominal, cardiac, vascular, and other major types of surgeries. The medical ICU also has 24 beds and their patients are in the intensive care for sepsis, pneumonia, respiratory failure, acute myocardial infarction, and other severe medical problems. This hospital's combined ICU urinary tract infections were historically below the 25th percentile benchmark of the NHSN quality indicator. However, nursing care is much different in the ICUs which have a lower patient to nurse ratio (1 or 2 patients to 1 nurse) than an acute care or progressive care units (4 or 5 patients to 1 nurse). A question that could be researched, but not explored in this study, is "Does staff to patient rato affect hospital acquired infections?

The only CAUTI rates available in the hospital were from the ICUs, and the data demonstrated 0.0 per 1000 urinary catheter days in the Surgical ICU and 0.6 per 1000 urinary catheter days in the Medical ICU. These CAUTI rates are below the NHSN benchmark. We did not have CAUTI rates for the PCUs, and did not want to assume that the rates were comparable. CAUTI rates should be reviewed to capture the quality of nursing practices in progressive units. Assessing and identifying the hospital CAUTI rates was crucial not only for patient safety but also to meet the newly announced CMS requirements on CAUTI reimbursement to the hospital. Consequently, this hospital decided to investigate the progressive care unit's CAUTI rate and evaluate a new evidence-based protocol on hospital acquired infections.

One of the four progressive care units was selected based on their increased incidence of urinary tract infections. This unit had a 41 patient bed capacity and various patient diagnoses that require telemetry monitoring.

Data was collected on the progressive care unit for seven consecutive days on a UTI Surveillance Log (see Figure 1), an existing form that was already in use by the hospital's Infection Prevention Department for tracking ICU rates. The data collected consisted of the patient's name, medical record number, admission date, infection identification date, patient's signs and symptoms related to infection, indwelling catheter insertion date, indwelling catheter discontinuation date, urine organism analysis, and antibiotic coverage. For consistency and quality control purposes, one member of the team collected all the data at the same time each day. 
Urinary Tract Infection SURVEILLANCE LOG

\begin{tabular}{|c|c|c|c|c|c|c|c|c|c|}
\hline Room & MRN & Resident Name & Adm Date & Inf. Date & Condition/Symptoms* $^{*}$ & Foley & Organism/Comments & Antibiotics & $\begin{array}{c}\text { ICP Use } \\
\text { Only }\end{array}$ \\
\hline & & & & & & Y N & & & \\
\hline & & & & & & Y N & & & \\
\hline & & & & & & Y N & & & \\
\hline & & & & & & Y N & & & \\
\hline & & & & & & YN & & & \\
\hline & & & & & & YN & & & \\
\hline & & & & & & YN & & & \\
\hline & & & & & & Y N & & & \\
\hline & & & & & & Y N & & & \\
\hline & & & & & & YN & & & \\
\hline & & & & & & YN & & & \\
\hline & & & & & & YN & & & \\
\hline & & & & & & YN & & & \\
\hline & & & & & & YN & & & \\
\hline & & & & & & Y N & & & \\
\hline \multicolumn{6}{|c|}{ "KEY: Place symptom \# in column for symptoms } & \multicolumn{3}{|c|}{11 positive urine culture, $>105 / \mathrm{cc}$ of urine with } & \\
\hline \multicolumn{3}{|c|}{1 Fever, Specity ${ }^{\circ} \mathrm{F}$ or ${ }^{\circ} \mathrm{C}$} & \multicolumn{3}{|c|}{6 Chills } & \multicolumn{3}{|c|}{ no more than two species of microorganisms. } & $\begin{array}{l}\text { ICP Use Only: } \\
\mathrm{N}=\text { New Case }\end{array}$ \\
\hline \multicolumn{3}{|c|}{2 Change in mentalfundamental status } & \multicolumn{3}{|c|}{7 Positive uninalysis } & \multicolumn{3}{|c|}{12 New burning pain on urination } & $\begin{array}{l}\mathrm{RC}=\text { Recurrent } \\
\mathrm{C}=\text { Acquired }\end{array}$ \\
\hline \multicolumn{3}{|c|}{3 organisms seen on Gram stain of unspun urine } & \multicolumn{3}{|c|}{8 frequency. } & \multicolumn{3}{|c|}{13 positive dipstick for leukocyte esterase and/or nitrate } & $\begin{array}{l}\text { autside focily } \\
\emptyset=\text { No infection }\end{array}$ \\
\hline \multicolumn{3}{|c|}{4 Change in character of urine(visual or by smell) } & \multicolumn{3}{|c|}{9 dysuria, } & \multicolumn{3}{|c|}{14 physician diagnosis of a urinary tract infection } & $\begin{array}{r}\text { by definition } \\
V=\text { Noted by icP }\end{array}$ \\
\hline \multicolumn{3}{|c|}{5 suprapubic tenderness } & \multicolumn{3}{|c|}{10 urgency, } & \multicolumn{3}{|c|}{15 physician institutes appropriate therapy for a urinary tract infection. } & \\
\hline
\end{tabular}

Figure 1. Urinary Tract Infection Surveillance

In addition to the UTI Surveillance Log a Catheter Associated Surveillance Denominator Log (see Figure 2) was completed. The data on the log included the number of patients on the unit and the number of patients with indwelling catheters. The progressive care unit averaged eighteen patients per day with urinary catheters. Once the data was collected the Infection Control Practitioner analyzed the data and compared it to the National Healthcare Safety Network (NHSN) for progressive care units of similar size to establish baseline data.

Prior to implementation of this project the CAUTI rate on the progressive care unit was above the 25th percentile of the National Healthcare Safety Network (NHSN) hospitals for acute care units. The device rate was above the 90th percentile of NHSN hospitals. The baseline data indicated that the hospital used catheters more frequently and kept them in place longer than $90 \%$ of other hospitals (see Figure 4).

\section{Review of literature}

The literature offers a substantial amount of evidence on the prevention of CAUTIs with six overall best practice recommendations: 1) utilizing silver alloy coated catheters, 2) preventing of unnecessary insertion, 3) limiting duration of the catheter use, 4) maintaining an aseptic catheter system, 5) providing good perineal hygiene, and 6) following proper hand hygiene ${ }^{[5]}$. We examined the hospital's clinical practice, guidelines of care, policies and products. The following is a brief overview of the six best practices as adopted by this hospital. 


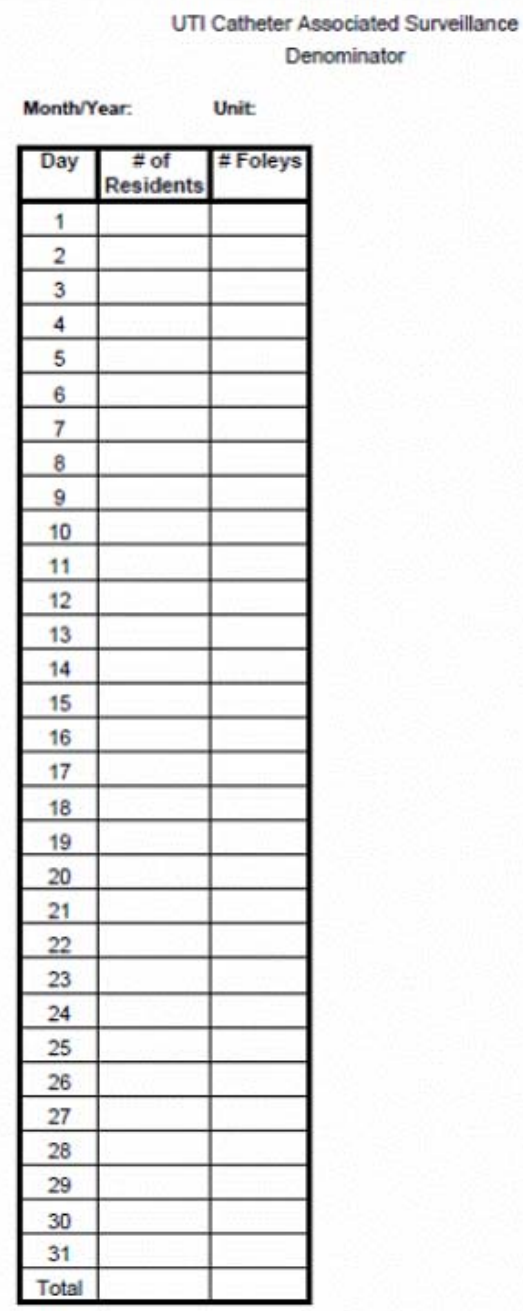

SMH-SCVMC-GH Form

Figure 2. Urinary Tract Infection Surveillance

\subsection{Silver-Alloy antimicrobial catheters}

Catheters have been manipulated by using various coatings and changes in raw material to try to prevent infection and improve comfort. Coatings such as hydrogels, silicone, and silver alloy have been used. A systematic review by Beattie and Taylor ${ }^{[6]}$ showed a consistent positive pattern with the use of silver-alloy urinary catheters to reduce catheter associated urinary tract infections. As a result of recent studies this hospital had instituted the use of silver alloy coated catheters in their indwelling catheter kits prior to this study.

\subsection{Preventing unnecessary insertion}

Despite our knowledge of the risks of developing a CAUTI, hospital personnel continue to place indwelling catheters routinely in hospitalized patients. Gokula et al. ${ }^{[1]}$ found an appropriate indication for catheterization in only 46 percent of elderly patients. The majority of patients were being routinely catheterized in the emergency department, many times without a physician's order. This practice should not occur since even with short term use, less than one week, catheter placement can result in complications. Complications noted in the ICUs include: urethritis, hematuria, urethral strictures, bladder perforation, and urosepsis which can be life threatening ${ }^{[7]}$. 
The use of indwelling urethral catheters should be limited to preoperative use for surgical procedures, urine output monitoring in critically ill patients, acute urinary retention, and to assist in pressure ulcer healing for incontinent patients ${ }^{[2]}$. In 2009, the Center for Disease Control (CDC) listed recommendations for appropriate use, and the examples given were consistent with those of Jacobsen. Inappropriate use might be to obtain a urine culture when the patient is able to void ${ }^{[8]}$. When a urinary catheter is placed, the healthcare workers need to ask the question, "Does this patient really need this catheter?" If there is not a valid reason for catheter insertion the nurses should question the reason for insertion.

\subsection{Timely removal of catheter}

The duration of catheterization is the most important risk factor for development of infection ${ }^{[9]}$. The risk of bacteriuria associated with catheter use increases 3 to 10 percent each day the catheter remains in place. It is estimated that 10 to 50 percent of patients having short term catheterization (less than 7 days) will develop a CAUTI ${ }^{[2]}$. Accountability for catheter removal is an important aspect of nursing practice and the nurse must question the physician whether the catheter is still necessary.

\subsection{Aseptic catheter system}

The importance of sterile technique is fundamental to the placement of intermittent or long term urinary catheters. Indwelling catheters must be placed using aseptic technique with sterile equipment. Maintaining a closed catheter system is crucial to reducing the risk of CAUTI ${ }^{[2]}$. The catheter and drainage bag are disconnected only when the catheter is irrigated. Urine specimen collection is performed using a sterile needle and syringe through the provided urine sampling port only after the port is cleaned with disinfectant.

\subsection{Perineal hygiene}

Patients that have indwelling catheters are at higher risk of CAUTI as a result of the direct line of microorganisms that migrate to the bladder along the outside of the catheter. Several resources suggest that the catheter insertion site be cleaned with soap and water daily when bathing or showering ${ }^{[10,11]}$.

\subsection{Proper hand hygiene}

A universal precaution, which includes hand hygiene, is extremely important in CAUTI prevention. This hospital developed a patient safety culture backed by administration to provide resources for several quality measures including hand hygiene education and ensure compliance by conducting audits. Healthcare workers must use the antiseptic hand gel or soap and water.

When emptying a catheter bag staff should clean their hands, don gloves, and clean hands again after removing their gloves. If patients empty their own drainage bags they need to be taught the importance of proper hand hygiene ${ }^{[7]}$. Renewed emphasis on hand hygiene for healthcare personnel will decrease the risk of cross contamination between patients.

The following interventions emerged as the key components of this program due to the overwhelming amount of evidence in the literature: utilizing silver alloy coated catheters, preventing unnecessary insertion, removing catheters in a timely manner, maintaining an aseptic catheter system, providing perineal hygiene, and adopting proper hand hygiene technique of caregivers. After review of this hospital's practices, it was noted that the most important focus should be recognition of appropriate use and timely removal of the urinary catheters.

\section{Methodology}

This evidence-based practice project utilized a pre-post design methodology. The implementation and design team consisted of a unit based nurse educator, a staff nurse, two student nurses, and the Director of Nursing Education, Research 
and Professional Development. The team met and each member was assigned articles to review critique and synthesize the review of literature. When the team met the second time in February of 2009 they discussed the summary of review of literature and designed the project. The team wanted to answer the following question: "Among progressive care patients with indwelling Foley catheters, does a new protocol for prevention of urinary tract infections (versus current practice), result in an overall decrease in the amount of hospital acquired urinary tract infections at the time of hospital discharge?” The team proceeded with the implementation of the project after receiving approval from the hospital's Institutional Review Board (IRB) and Administrative Review Committee.

\section{Education of evidence-base practice}

A PowerPoint presentation was developed summarizing the best practices found in the literature. A poster illustrating key points was created and taken to the unit by one of the team members and used as a visual aid when conducting education to the progressive care unit's staff. Education was accomplished at staff meetings, unit practice council meetings, and a one on one in-service with nurses on the progressive care unit. Education reinforced the use of silver alloy coated catheters, regular perineal care, maintaining an aseptic system, proper hand hygiene, and regular emptying of the urinary drainage bag. The poster remained on the unit in a prominent place for staff reference.

Education was provided to all nursing staff, nursing assistants, and physicians. Preventing unnecessary urinary catheter use and timely removal was necessary to the success of this project. An Indwelling Catheter Visual Prompt Tool was developed to support prevention efforts. The purpose of the tool was to remind the physician and nursing staff of catheter placement and was located in the front of the chart (see Figure 3). The tool listed appropriate indications for continued catheter use and requested the physician to write a daily order discontinuing the catheter or documentation of the reason for the catheter's continuance. After several weeks of use, the nurses recommended moving the tool to the progress notes where the physician documents daily to increase physician compliance.

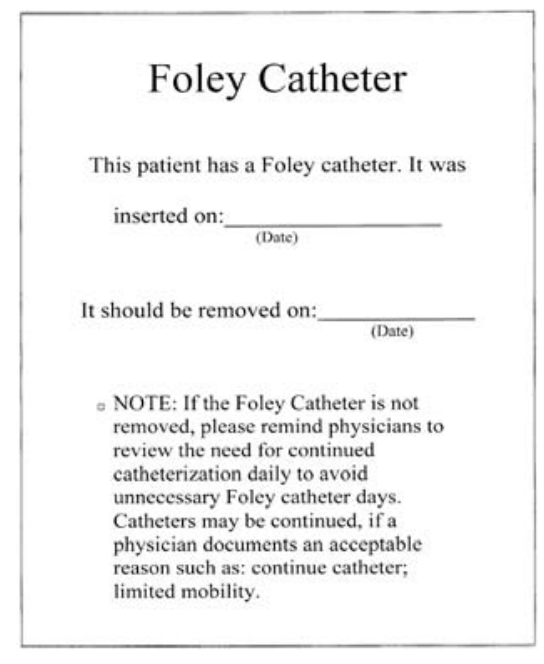

Figure 3. Visual Prompt

Education encompassed the complications that may occur in patients with indwelling catheters and the importance of discontinuation as soon as possible. Fundamentals of indwelling catheter nursing care and aseptic urine sampling was the focus of the education. Nurses were reminded to maintain sterility when performing a catheterization and the nursing assistants were reminded to perform proper hand hygiene and maintain a closed sterile system when emptying the drainage bags. It was important to include the nursing assistants since they performed the majority of perineal care and emptied the urinary drainage bags. 
A two-page colorful handout was created titled "Guidelines to Decrease Our CAUTI Rate”. The handout provided appropriate indications for insertion of indwelling catheters. Indications included diagnostic evaluation, therapeutic short term (less than 1 week), therapeutic long term (greater than 4 weeks), uncorrectable bladder outlet obstruction, recalcitrant decubitus ulcers, neurogenic bladder, or palliative care in terminally ill patients. Reasons such as the patient having decreased mobility or for healthcare workers convenience were not acceptable. The healthcare team was introduced to the visual prompt and its use. The education continued for approximately three weeks and reached approximately $80 \%$ of the nursing staff.

Cost to this project was budget neutral. All staff involved and staff education occurred during work time. Educational materials such as handouts and poster boards were absorbed with daily operations.

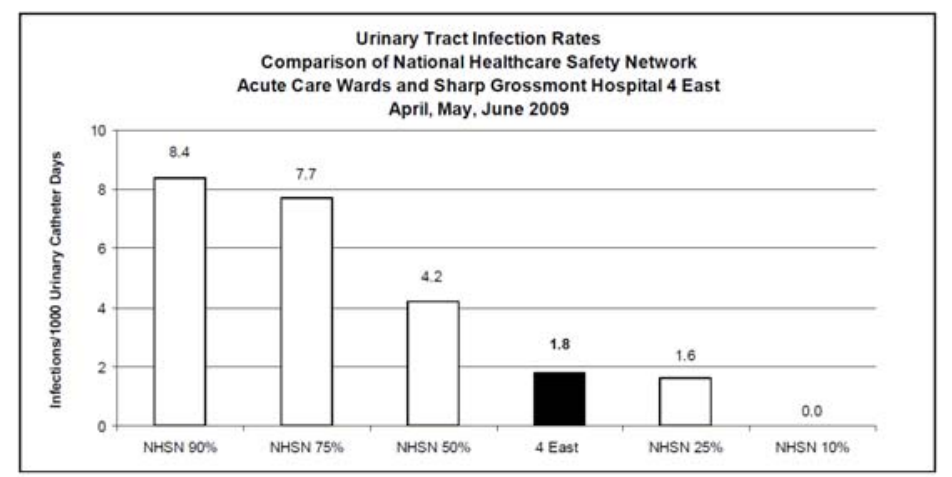

Figure 4. Urinary Tract Infection Rate Pre Implementation

\section{Results}

Analysis of the data was performed by the Infection Prevention Practitioner as to whether any patients met the National Healthcare Safety Network (NHSN) criteria for CAUTI. Baseline data on the point prevalence study prior to the implementation of the evidence-based practice project revealed that the CAUTI rate was 1.8/1000 catheter days and the device utilization was 0.5 (device days/patient days). Five months after the implementation of the project the point prevalence study was repeated. The infection rate decreased to $0.7 / 1000$ catheter days and catheter utilization decreased to 0.3 (device days/per patient days) (see Figure 5 and Table 1). There was no statistical analysis conducted on these rates.

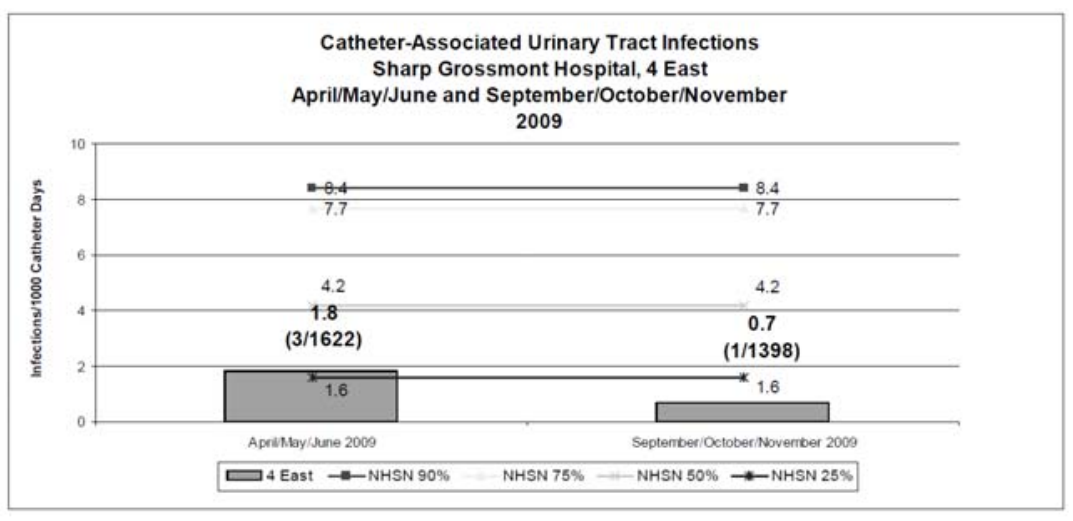

Figure 5. Urinary Tract Infection Rate Pre \& Post Implementation 
Table 1. Point Prevalence Study

\begin{tabular}{|c|c|c|}
\hline & April/May/June 2009 Quarter & $\begin{array}{l}\text { September/October/November } 2009 \\
\text { Quarter }\end{array}$ \\
\hline Number of days of data collection & 5 & 7 \\
\hline $\begin{array}{l}\text { Catheter-associated urinary tract } \\
\text { infections }\end{array}$ & 3 & 1 \\
\hline Device (catheter) days & 102 & 77 \\
\hline Patient days & 222 & 279 \\
\hline $\begin{array}{l}\text { Infection rate/ } 1000 \text { catheter days for the } 5 \\
\text { day period only }\end{array}$ & 29.4 & 12.9 \\
\hline Device utilization & $\begin{array}{l}0.5 \\
\text { (102 catheter days/ } \\
222 \text { patient days) }\end{array}$ & $\begin{array}{l}0.3 \\
\text { (77 catheter days/ } \\
279 \text { patient days) }\end{array}$ \\
\hline CAUTI rate & $1.8 / 1000$ & $0.7 / 1000$ \\
\hline
\end{tabular}

\section{Discussion}

After implementation of this project the CAUTI and device rates were less than 25th percentile of the NHSN hospitals suggesting that the project was successful in decreasing catheter days. Several studies have shown that decreased catheter days relate directly to decrease catheter related urinary tract infections. Salamon (2009) revealed that decreasing catheter days could be accomplished by educating nurses about catheter awareness and making catheter removal a priority. This hospital's device rate decreased by 20 percent.

Education sessions increased both physician and nurse awareness of appropriate indications for indwelling catheterization and suggest that decreased catheter days do relate to decreased CAUTIs. Having physician support was crucial to the success of this project and initially some physicians were resistant to comply with documentation for continued catheter use. The director and the Chief Nursing Officer appealed to the physicians in their monthly executive meeting to capitulate to this new protocol. In addition results of the project were shared at the Quality Council where physicians, the Chief Medical Officer, and Chief of Staff were present. Discussion was held as to how best to continue the new practice. In time the physicians and nurses understood that this initiative was imperative to quality patient care thus adopting this new practice.

\section{Limitations}

There are several limitations in this project. The focus of this project was to implement best practice regarding the prevention of CAUTI. Although an evidence-based practice methodology was utilized it was not as rigorous as a research project. For example this project was conducted at one hospital and on only one of the four progressive care units. This resulted in patients with similar proportions of diagnoses. A medical unit might have shown very different results. Another limitation was the lack of a champion on the progressive care unit.

To sustain the projects efforts several strategies should be considered. Many change projects enlist a staff member who becomes the project coordinator or champion of the project. Having an effective champion on a unit to spearhead the project could increase the effectiveness of the project. The nurse champions are recruited from individual units to ensure that continued education occurs as needed and can participate in audits to check for compliance. In this project no nurse champions were identified. However, the majority of the unit's nurses were accepting of this initiative and their enthusiasm increased with the knowledge that their patient's outcomes improved. 
Another common strategy is to include continuing education as part of the program. Regularly schedule classes or in-services will assist in the sustainability of the program's content. Lastly, recurrent prevalence studies with data on device days and infection rates will provide feedback on staff adherence to best practice.

\section{Conclusion}

The purpose of this evidence-based practice project was to decrease the CAUTI rate in the progressive care unit by implementing a new CAUTI protocol. This project was a success as evident by the decreased CAUTI rates and decreased device days. By creating a program using the six guidelines from a literature review and educating the staff on these key clinical practices a hospital can decrease catheter utilization and infection rates.

Due to the success of the project the decision was made to expand this evidenced based intervention to the rest of the hospital's progressive care and acute care units. Education will be accomplished by unit educators and clinical nurses under the direction of the progressive care Clinical Nurse Specialist. Several educators from various acute care and progressive care units will serve as champions to support the implementation of this intervention. Focus of the hospital wide project will consist of 1) formation of a CAUTI task force, 2) employing CAUTI Champions, 3) education on the evidence-based practices clinical recommendations, 4) use of the visual prompt, and 5) prevalence quarterly data collection.

\section{References}

[1] Gokula RM, Smith MA, Hickner J. Emergency room staff education and use of a urinary catheter indication sheet improves appropriate use of Foley catheters. Am J Infect Control. 2007; 35(9): 589-593. PMid:17980237 http://dx.doi.org/10.1016/j.ajic.2006.12.004

[2] Jacobson SM, Stickler DJ, Mobley HL, Shirtliff ME. Complicated catheter-associated urinary tract infections due to Escherichia coli and Proteus mirabilis. Clin Microbiol Rev. 2008; 21(1): 26-59. PMid:18202436 http://dx.doi.org/10.1128/CMR.00019-07

[3] Lawrence EL, Turner IG. Materials for urinary catheters: a review of their history and development in the UK. Med Eng Phys. 2005; 27: 443-453. http://dx.doi.org/10.1016/j.medengphy.2004.12.013

[4] Salamon, L. Catheter-associated urinary tract infections: a nurse-sensitive indicator in an inpatient rehabilitation program. J Rehabil. 2009; 34(6): 237-241.

[5] Agency for Healthcare Research and Quality (AHRQ). Pub. No. 08-0043-CD. April 2008.

[6] Beattie M, Taylor J. Silver alloy vs. uncoated urinary catheters: a systematic review of the literature. J Clin Nurs. 2011; 20(15-16): 2098-2108. PMid:21418360 http://dx.doi.org/10.1111/j.1365-2702.2010.03561.x

[7] Reilly L, Sullivan P, Ninni S, Fochesto D, Williams K, Fetherman, B. Reducing foley catheter device days in an intensive care unit. AACN Adv Crit Care. 2006; 17(3): 272-283. PMid:16931923 http://dx.doi.org/10.1097/01256961-200607000-00007

[8] Buchmann B, Stinnett G. Reducing rates of catheter-associated urinary tract infection. Ala Nurse. June, July, August, 2011 ; 5. PMid:21853722

[9] Lo E, Nicolle L, Classen D, et al. Strategies to prevent catheter-associated urinary tract infections in acute care hospitals. Infect Control Hosp Epidemiol. 2008; 29(1): 41-50. PMid:18840088 http://dx.doi.org/10.1086/591066

[10] Centers for Disease Control and Prevention. The National Healthcare Safety Network Protocols and Definitions DeviceAssociated module. http://www.cdc.gov/nhsn/PDFs/slides/CAUTI.pdf. Accessed Dec 28. 2011.

[11] Willson M, Wilde M, Webb L, et al. Nursing interventions to reduce the risk of catheter-associated urinary tract infection. Wound Ostomy Continence. 2009; 36(2): 137-154. PMid:19287262 http://dx.doi.org/10.1097/01.WON.0000347655.56851.04

[12] Godfrey H, Fraczyk L. Preventing and managing catheter associated urinary tract infections. Br J Community Nurs. 2004; 10(5): 205-212. 